\title{
Un examen del trilema de Akeel Bilgrami desde el externismo social*
}

\section{A review of Akeel Bilgrami's trilemma from social externalism}

\author{
SILVIA ANDRÉS BALSERA \\ Universidad Complutense de Madrid
}

Recibido: 22-02-2010 Aprobado definitivamente: 22-07-2010

\section{RESUMEN}

El propósito de este artículo es defender que el externismo social de Burge puede enfrentarse con éxito al trilema de Bilgrami, un argumento que pretende mostrar la incompatibilidad entre el externismo ortodoxo, dentro del cual figura el externismo de Burge, y el acceso privilegiado a los propios contenidos mentales. Además, pretendemos poner de manifiesto la existencia de ciertas tensiones en la versión del externismo defendida por Bilgrami.

\section{PALABRAS CLAVE \\ ATRIBUCIONES CONCEPTUALES, CONTENIDO PSICOLÓGICO, INTERNISMO, EXTERNISMO}

\begin{abstract}
The aim of this paper is to defend that Burge's social externalism can successfully face Bilgrami's trilemma, an argument that intends to show the incompatibility between orthodox externalism, in which Burge's externalism is featured, and the privileged access to our own mental contents. In addition, we mean to demonstrate the existence of certain tensions in Bilgrami's view of externalism.

KEYWORDS

CONCEPTUAL ATRIBUTIONS, PSYCHOLOGICAL CONTENT, INTERNALISM, EXTERNALISM

* Este trabajo ha sido realizado en el marco del proyecto FFI2008-03092 financiado por el Ministerio de Ciencia e Innovación. Agradezco al revisor anónimo sus observaciones y al profesor Luis Fernández Moreno sus valiosos comentarios sobre el borrador de este artículo.
\end{abstract}

(C) Contrastes. Revista Internacional de Filosofía, vol. XVI (2011), pp. 49-66. ISSN: 1136-4076 Departamento de Filosofía, Universidad de Málaga, Facultad de Filosofía y Letras

Campus de Teatinos, E-29071 Málaga (España) 


\section{INTRODUCCIÓN}

LA PREGUNTA POR LOS FACTORES que determinan esencialmente los conceptos y contenidos mentales de los individuos es una cuestión que ha preocupado sobremanera a los filósofos de la mente en las últimas décadas. El aspecto más discutido de este problema tiene que ver con la suficiencia (o no) de los elementos internos a los individuos a la hora de especificar los contenidos de sus actitudes psicológicas. ${ }^{1} \mathrm{Si}$ la determinación del contenido es puramente interna, como defienden los partidarios del denominado internismo psicológico, entonces dos individuos internamente idénticos pensarán, a fortiori, lo mismo. Muchos autores, sin embargo, han elaborado argumentos para mostrar la necesidad de contar con ciertos elementos externos al sujeto entre las condiciones de identidad de sus contenidos psicológicos. Aquellos que optan por esta segunda opción reciben el nombre de «externistas». No obstante, el externismo, como posición teórica contrapuesta al internismo, dista de ser una tesis unitaria. Así, en este trabajo abordaremos la sugerencia de Akeel Bilgrami de distinguir dos clases de externismo, a saber, el externismo ortodoxo, una de cuyas versiones es el externismo social de T. Burge, y un externismo más débil, no comprometido con la teoría causal de la referencia, del cual se declara partidario el propio Bilgrami. En varias de sus obras ${ }^{2}$ este autor desarrolla una estrategia en forma de trilema para socavar la plausibilidad del externismo ortodoxo, al mismo tiempo que intenta demostrar que su propia comprensión del externismo no adolece del mismo defecto que se le imputa a la variante ortodoxa y que constituye el centro de gravedad del trilema, a saber, su incompatibilidad con la autoridad que se presupone en el conocimiento que los individuos tienen de sus propios contenidos mentales.

Por lo que a nosotros respecta, en primer lugar intentaremos disminuir la eficacia de los argumentos que Bilgrami ofrece para colocar a la variante social del externismo ortodoxo en la tesitura de tener que enfrentarse a su trilema. En segundo lugar, mostraremos que la propuesta externista de Bilgrami no está exenta de ciertas tensiones internas y se inclina peligrosamente hacia una concepción meramente instrumentalista de lo mental.

1 A modo de aclaración terminológica, entendemos por actitud psicológica o pensamientos las actividades mentales de los individuos dirigidas hacia un contenido proposicional. Así, son actitudes psicológicas creer, temer, buscar, desear, etc., que $\mathrm{P}$, donde $\mathrm{P}$ es un enunciado que especifica el contenido de cada una de estas actitudes. Consideraremos, como es usual, que la identidad de una actitud es función de su contenido y que, a su vez, la identidad de un contenido es función de los conceptos que involucra.

2 Nos referimos a Bilgrami 1992a, 1992b y 2003. 


\section{LOS EXTERNISMOS ORTODOXOS Y EL TRILEMA DE BILGRAMI}

Según hemos dicho, las propuestas de Bilgrami parten de una distinción entre distintas variantes del externismo psicológico, así que nuestra primera tarea es ofrecer una enunciación del contenido de cada una de estas versiones. En primer lugar, este autor establece que el externismo en general ha de comprenderse como una tesis que niega la independencia de los contenidos mentales del individuo respecto del mundo externo. Esta comprensión del externismo, que denominaremos, con Bilgrami, «externismo general» (1992b, p. 364), constituye más bien el común denominador de todas las variantes del externismo, por cuanto que su contenido establece meramente la negación del modo internista de individuación de los contenidos mentales. ${ }^{3}$

A partir de esa definición de mínimos se configuran las propuestas externistas más usuales, que Bilgrami denominará «externismos ortodoxos», dentro de los cuales encontramos el externismo social de Burge y el externismo natural de Putnam. En cuanto a este último, que Bilgrami denomina también externismo «esencialista-causal», mantiene que ciertos términos, los términos de género natural, se refieren directamente (sin mediación conceptual) a objetos que comparten una determinada naturaleza (Bilgrami 1992a, pp. 26-26). Con respecto al externismo social de Burge, que es el que ocupará principalmente nuestro interés en este trabajo, Bilgrami describe su núcleo teórico diciendo que «las prácticas lingüísticas de una comunidad a menudo afectan a la mente individual en el sentido en que atribuimos a un individuo los mismos conceptos que a sus semejantes, aun cuando tenga creencias bastante divergentes»> (1992a, p. 23).

La mejor manera de profundizar en la comprensión del externismo social consiste en acudir al experimento mental que el propio Burge ofreció a favor de su tesis en su artículo de 1979. Dicho experimento tiene dos partes, ${ }^{4}$ la primera de las cuales presenta el caso de un hablante del mundo actual, llamémosle Bert, que posee una serie de creencias en torno a la artritis. Por ejemplo, Bert cree (con verdad) que padece artritis, que es mejor tener artritis que tener cán-

3 El mínimo externista consiste en su presunta incompatibilidad con el escenario cartesiano del genio maligno y sus versiones contemporáneas. Cf. Bilgrami 1992b, p. 385.

4 Fodor, en su obra de 1987, p. 28, afirma que en los experimentos mentales de Burge se ponen en juego dos intuiciones, que se corresponden con las dos partes que hemos distinguido nosotros. Loar, en su artículo de 1985, p. 181, segmenta del mismo modo el experimento de Burge, argumentando que la primera parte de este experimento sirve para demostrar que no es necesario que dos sujetos asocien el mismo rol conceptual a un término (internamente determinado) para que se les pueda adscribir el mismo contenido psicológico. En la segunda parte, Burge alegaría que la identidad de rol conceptual tampoco es una condición suficiente para atribuir los mismos contenidos. 
cer, que la artritis es una enfermedad muy dolorosa y, además, cree que tiene artritis en el muslo. Esta última creencia es, sin embargo, errónea, puesto que la artritis es una dolencia que afecta sólo a las articulaciones y es de suponer que si Bert decidiera comentar sus temores con un médico éste le diría que es imposible que su dolor de muslo se deba a la artritis. La idea de Burge es que parece intuitivo suponer que tanto Bert como su médico comparten el concepto de artritis, aun cuando, por hipótesis, sus respectivos grados de competencia en el dominio de dicho concepto difieren notablemente. La segunda parte del experimento mental tiene como protagonista a un hablante contrafáctico, Bert*, que es idéntico en todos sus aspectos internos a Bert, el individuo del mundo actual. No obstante, en el entorno de Bert* el término «artritis» se aplica tanto a enfermedades de las articulaciones como a ciertos trastornos reumatoides. Si Bert* acudiera a un médico para comunicarle su sospecha de que padece artritis en el muslo éste considerará su temor perfectamente razonable. Dicho esto, la pregunta más interesante desde el punto de vista de Burge es la siguiente: ¿atribuiríamos a este individuo, Bert*, creencias acerca de la artritis? Parece difícil afirmar que Bert* posee ese concepto, pues en su entorno lingüístico mediante el término «artritis» los hablantes se refieren, por hipótesis, a una enfermedad distinta.

La moraleja que extrae Burge de este escenario establece que los contenidos mentales que poseen los individuos dependen de las prácticas lingüísticas de su entorno externo, de manera que, por un lado, dos individuos, como Bert y su doctor, cuyas creencias difieren (y son, por ello, individuos internamente distintos) pero que pertenecen a una misma comunidad lingüística, poseen el mismo concepto y, por otro, dos individuos internamente idénticos pero que están insertos en diferentes entornos lingüísticos, como Bert y Bert*, poseen, sin embargo, conceptos distintos.

Para Bilgrami, el externismo de Putnam y el externismo de Burge pueden englobarse bajo el rótulo de «externismos ortodoxos» en tanto que las propuestas de ambos tienen un fondo teórico común, a saber, la teoría de la referencia directa, defendidas por Kripke (1972) y Putnam (1975). ${ }^{5}$ Según estas teorías, nuestros significados y conceptos vienen especificados en términos de (las na-

5 Cabe discutir la comprensión de Bilgrami de la teoría de la referencia directa. En nuestra opinión, bajo este rótulo se engloban dos tipos de propuestas, a saber, aquellas que rechazan que la referencia venga determinada por un sentido descriptivo al modo de Frege (Putnam y Kripke se adhieren claramente a esta versión de la teoría) y aquellas concepciones que mantienen que la contribución semántica de ciertos términos a la proposición en la que figuran es únicamente su referente (es discutible que Putnam suscriba esta opinión pero parece que Kripke aboga por ella en algunas ocasiones). En lo que sigue, asumiremos la caracterización de la teoría de la referencia directa que propone Bilgrami, dejando claro que constituye una fusión de las dos propuestas que distinguimos. 
turalezas objetivas o esencias que constituyen) su extensión y ello ocurre aunque nosotros desconozcamos (las naturalezas objetivas o esencias que constituyen) la extensión de estos conceptos. Además, el éxito de un acto de referir que involucra el uso de los términos lingüísticos así especificados tiene lugar sin necesidad de mediaciones conceptuales por parte del hablante. Con respecto al externismo ortodoxo de Burge, Bilgrami nos dice que son los expertos, un conjunto especialmente competente de hablantes, los que determinan la extensión de los conceptos, cuya identidad viene especificada, al igual que para Putnam, en función de dicha extensión. Aquellos individuos que mantienen una actitud deferente hacia esos expertos, en virtud de su pertenencia al entorno linguístico en el cual esos hablantes son expertos, poseerán los mismos conceptos que estos, aun cuando ignoren cuáles son las reglas de aplicación de los términos lingüísticos que expresan los conceptos y que nos proporcionarían su referente. ${ }^{6}$

Una vez que hemos recogido lo fundamental de las propuestas externistas en su variante ortodoxa, vamos a reconstruir el argumento que Bilgrami desarrolla en forma de trilema con el fin de mostrar que el externismo ortodoxo es prima facie incompatible con el conocimiento privilegiado que cada uno de nosotros tenemos sobre nuestros propios contenidos mentales. ${ }^{7}$ La idea fundamental de ese argumento consiste en que los presupuestos del externismo ortodoxo legitiman la atribución de pensamientos inconsistentes a los individuos. Pensemos que la teoría causal de la referencia, situada a la base de los externismos ortodoxos, según dice Bilgrami, considera que la esencia científica o naturaleza objetiva del referente es suficiente para determinar los significados y conceptos expresados por términos como «artritis» o «agua». Volvamos al caso de Bert, el protagonista del experimento mental de Burge, quien creía padecer artritis en el muslo. Bilgrami afirma que si damos la razón a Burge y atribuimos a Bert el concepto de artritis, que es el concepto que indiscutiblemente posee su médico, entonces estaremos realizando una atribución muy poco caritativa, en la medida en que el concepto de artritis es esencialmente el concepto de una enfermedad que afecta únicamente a las articulaciones. Dicho de otra manera, si sostenemos que Bert y el doctor comparten el concepto de artritis ello supondría atribuir a Bert la creencia de que padece en su muslo una enfermedad que afecta únicamente a las articulaciones, lo cual es una evidente inconsistencia. ${ }^{8}$ No obstante, los partidarios del externismo ortodoxo pueden esquivar esta acusación si aceptaran que los individuos como

6 Si Bilgrami denomina visión «causal-esencialista» al externismo de Putnam, al externismo de Burge lo califica de visión «científico-esencialista» (Bilgrami 1992b, p. 389).

7 Bilgrami aclara que su trilema muestra la incompatibilidad entre externismo y el conocimiento privilegiado de primera persona es una incompatibilidad prima facie porque no necesita apelar a viajes fantásticos entre entornos como los casos de traslado planteados por Burge en su artículo de 1982 y popularizados por Boghossian en sus escritos de 1992 y 1994.

8 Un argumento similar puede verse en Segal en 2000, pp. 65 ss. 
Bert, aunque perfectamente racionales, ignoran el significado completo de sus términos y conceptos. Esto es, Bert podría tener el pensamiento de que padece artritis en el muslo aunque no supiera que la artritis es una enfermedad que no afecta a los músculos. Sin embargo, desde los presupuestos del externismo ortodoxo es crucial para el concepto de artritis que sea una dolencia de las articulaciones, por lo que Bert tendría un pensamiento («tengo artritis en el muslo») que involucra conceptos que él desconoce de una manera fundamental. Como puede verse, esta estrategia para evitar las atribuciones de inconsistencias supone socavar la intuitiva autoridad del conocimiento de primera persona al afirmar que los individuos pueden ignorar los conceptos involucrados en sus creencias. Bilgrami reubica las posibles respuestas a este desafío como las dos primeras premisas de un trilema. $\mathrm{O}$ bien asumimos la legitimidad de ciertas atribuciones mentalistas inconsistentes, como parece desprenderse del externismo ortodoxo, o bien declaramos que un sujeto puede ignorar el contenido de sus propios pensamientos. Sin embargo, cabe una tercera posibilidad, correspondiente a la última premisa del trilema, que consiste en «bifurcar» el contenido psicológico y alegar, así, que los individuos tienen pleno conocimiento de su contenido mental, entendido como contenido estrecho o internamente determinado, pero que desconocen lo que concierne al contenido amplio que recibe una determinación externista. No obstante, el propio Burge aboga por una concepción unitaria del contenido y considera, por tanto, que la tercera opción del trilema no es viable (2007, p. 11). Por tanto, de rechazar esta última alternativa, Burge tendría que escoger entre conservar el acceso privilegiado y renunciar al externismo o seguir siendo externista y menoscabar seriamente la capacidad del sujeto para conocer sus propios contenidos mentales.

Una vez que Bilgrami ha expuesto los problemas que suscita la concepción ortodoxa del externismo psicológico, pasa a enunciar el contenido de su propia opción externista que viene recogida en lo esencial por la denominada «restricción (C)»: Al fijar un concepto externamente determinado de un agente, uno debe hacerlo buscando las proferencias indéxicamente formuladas del agente que expresan contenidos indéxicos que contienen ese concepto y escogiendo después el determinante externo del concepto que está en consonancia con otros contenidos que han sido fijados para el agente. ${ }^{9}$ Esta restricción nos ofrece el núcleo del externismo de Bilgrami. Por un lado, se apela al mundo externo en la fijación de los contenidos de un agente por la vía de las proferencias que contienen términos indéxicos, cuya dependencia semántica respecto del entorno externo parece indiscutible. Por otro lado, la atribución de contenidos inconsistentes queda cortocircuitada de raíz desde el momento en que se exige que las atribuciones sean coherentes con los contenidos que el agente ya po- 
see. Esta coherencia queda reforzada atendiendo a la distinción que establece Bilgrami entre los niveles agregativo y local de la atribución de conceptos. ${ }^{10}$ En el nivel agregativo, el nivel en el que operan las teorías del significado, hemos de situar todas las creencias que un agente asocia con un determinado término en calidad de creencias determinantes del concepto expresado mediante el uso de dicho término. Es de suponer que en este nivel los individuos no compartirían nunca un concepto, puesto que para ello se necesitaría que compartiesen absolutamente todas las creencias que le conciernen. No obstante, el nivel agregativo no es aquél en el que tienen lugar nuestras explicaciones de las acciones que llevan a cabo los agentes, sino un segundo nivel, el local. Bilgrami entiende que son las distintas localidades o contextos los que determinan qué creencias son relevantes para atribuir un concepto a un individuo. De esta manera, determinaremos si un individuo posee o no un concepto en función de si posee (únicamente) aquellas creencias que la localidad en la que tiene lugar la atribución hace relevantes.

En suma, la opción externista por la que aboga Bilgrami tiene como objetivo evitar las atribuciones no caritativas que él imputa al externismo ortodoxo al tener en cuenta que los pensamientos relevantes para realizar las atribuciones mentalistas vienen determinados por la localidad en la que se llevan a cabo.

\section{REFERENCIA DIRECTA, REPRESENTACIONES Y ATRIBUCIÓN DE CONCEPTOS}

En esta sección buscaremos precisar el alcance del compromiso del externismo social propugnado por Burge con la teoría de la referencia directa, tal y como la concibe Bilgrami, con el objeto de debilitar en lo posible la argumentación que éste desarrolla para establecer su trilema. Esta tarea tiene una importancia crucial, puesto que dicha argumentación gira en torno al presunto apoyo del externismo social en esa comprensión de la referencia, la cual propone determinar los significados y conceptos que los agentes poseen en función de las naturalezas y esencias de sus referentes y ello sin que se requiera ningún tipo de mediación cognitiva, sólo vínculos de tipo causal (Bilgrami 1992a, p. 25). Si esto es así, dice Bilgrami, podríamos atribuir a los agentes conceptos completamente desnudos de contenido representacional asociado, por lo que las atribuciones no caritativas serían un hecho (sólo evitables al precio de renunciar al auto-conocimiento). Así pues, este autor reconoce explícitamente que su justificación para establecer la primera premisa del trilema consiste en el compromiso del externismo ortodoxo con una teoría de la referencia directa teñida de esencialismo (1992b, p. 371).

10 Cf. pp. 386-387 en 1992 b y pp. 141 ss. en 1992a. 
Sin embargo, como decimos, cabe preguntarse cuál es el peso efectivo de ese compromiso en el caso del externismo de Burge. Para responder a esta cuestión sería recomendable acudir a las propias declaraciones de este autor al respecto, quien reconoce que sus primeros trabajos se vieron impulsados por la «revolucionaria» teoría de la referencia propuesta por Donnellan, Kripke y Putnam, aunque después él decidió tomar una nueva dirección para ocuparse de principalmente de la mente (más que del lenguaje) y de los modos de representación mental (Burge 2007, p. 7). Por otro lado, cuando Burge explica en qué consiste la afirmación fundamental del anti-individualismo, que es la denominación que él otorga a su propia concepción del externismo psicológico, suele hacer hincapié en el hecho de que la contribución de los factores del entorno externo es una contribución sólo de carácter parcial. Dicho de otra manera, el externismo de Burge no legitima prácticas «agresivas» de atribución, entendiendo por tales aquellas adscripciones de contenido mental que pasarían completamente por alto los modos de representación individuales. Por el contrario, Burge considera que es por lo general necesario (pero no suficiente) contar con factores que pueden rebasar o completar la perspectiva individual aunque sin violentarla. Para justificar esto, volvamos al caso de Bert. Este individuo no es un completo ignorante respecto de la artritis y así lo plantean las premisas del experimento mental: está en lo cierto al creer que tiene artritis y al pensar que se trata de una enfermedad muy dolorosa aunque preferible al cáncer. Cuando Bert dice a su médico ${ }^{11}$ «tengo artritis en el muslo» la ignorancia relativa que trasluce esta afirmación no es comparable con el desconocimiento absoluto respecto del concepto de verde que evidenciaría una afirmación como «el verde es un color delicioso» por parte de un segundo individuo a quien llamaremos Pedro. ${ }^{12}$ Esto, además, aporta plausibilidad a la intuición de Burge de atribuir al doctor y a Bert el mismo concepto de artritis, al contrario de lo que ocurriría en el caso de Pedro y su interlocutor, hablante competente del castellano, con respecto al concepto de verde. Estas observaciones pretenden moderar y matizar el alcance del compromiso que Bilgrami detecta entre las tesis de Burge y una teoría de la referencia que haría prescindible cualquier tipo de material cognitivo para establecer la referencia de nuestros términos lingüísticos.

Por otro lado, podríamos preguntarnos si las ideas de Burge se ven afectadas por los argumentos de Bilgrami contra una teoría de los conceptos puramente desentrecomilladora. Así, Bilgrami prevee una posible estrategia del externista ortodoxo para escapar al trilema, estrategia que consiste en apelar a una teoría desentrecomilladora, de acuerdo con la cual podemos especificar

11 Otra señal de que Bert no es un ignorante respecto de la artritis es que acude a un experto relevante, su médico, para resolver sus dudas que afectan a la aplicación de ese concepto.

12 Descartados los lapsus o accidentes psicológicos semejantes. 
el concepto expresado por el término «artritis» diciendo que ese concepto es meramente el concepto de artritis, sin que sean necesarias ulteriores redescripciones de esa noción (Bilgrami 1992a, pp. 43 ss. y 1992 b pp. 373 ss.). En efecto, el externista que optara por emplear este recurso podría esquivar gracias a él la primera premisa del trilema, puesto que en ese caso no tendría sentido reformular el concepto de artritis mediante la descripción que, según Bilgrami, captura su esencia científica y que constituye la base de la inconsistencia, a saber, la descripción de la artritis como una enfermedad que afecta únicamente a las articulaciones (1992a, p. 390). Sin embargo, tiene razón Bilgrami cuando replica que una teoría de esta clase sólo podría impedir las redescripciones al precio de inefabilizar los conceptos, vinculados de manera «directa» con su referente, en virtud de cadenas causales, y declarando prescindible cualquier tipo de componente representacional asociado al término relevante. Ahora bien, hemos visto que el anti-individualismo de Burge no respaldaría una comprensión desentrecomilladora de los conceptos, puesto que este autor sostiene que la determinación externista es sólo una parte, generalmente necesaria pero no suficiente, de la individuación conceptual y que las relaciones causales involucradas en esa determinación «no tienen que ser tan simples como los ejemplos sugieren [sino que p] ueden ser bastante complejas e indirectas»» (Burge 2007, p. 3).

No obstante, además de la propuesta desentrecomilladora, Bilgrami cuestiona un recurso genuino del anti-individualismo de Burge encaminado a impedir las atribuciones de pensamientos inconsistentes: el recurso que estriba en distinguir entre conceptos y explicaciones conceptuales (1989a, p. 282). Así, según Burge, son «explicaciones conceptuales» aquellas especificaciones reflexivas que podría proporcionarnos un individuo sobre el valor cognitivo de un determinado concepto, especificaciones en las que haría uso de otros conceptos relacionados y de inferencias a partir de experiencias perceptivas (1989a, p. 284). Bilgrami responde a Burge que tras esta distinción sólo se esconde una bifurcación de contenido basada en una bifurcación previa de conceptos. En base a ello, prosigue Bilgrami, «ahora se nos dice que hay dos nociones de conceptos 1) conceptos propiamente dichos, dados por la referencia externista y 2) conceptos en el sentido de explicaciones conceptuales que el agente puede articular bajo reflexión» (1992b, p. 370), de manera que mediante la apelación a esta segunda clase de conceptos podemos evitar inconsistencias. Ahora bien, no estamos de acuerdo con Bilgrami cuando califica a la distinción de Burge de bifurcación solapada. Y nuestro argumento tiene que ver con las consideraciones precedentes, en la medida en que sólo sería posible hablar de bifurcaciones en sentido propio si considerásemos que la determinación externista de los conceptos es equivalente a una práctica de atribución de contenidos «agresiva», que atiende sólo a los vínculos causales individuo-entorno en el 
sentido más craso, y que se abstiene por completo de tomar en consideración la perspectiva cognitiva del individuo. Pero para Burge esta equivalencia no es admisible. Cuando este autor afirma que dos individuos pueden ofrecer distintas articulaciones conceptuales si se les solicita una explicación acerca de lo que entienden por un mismo concepto no está separando radicalmente ni bifurcando las explicaciones conceptuales de los conceptos mismos. Una separación tal sólo tendría lugar si bastara con que el individuo estableciera vínculos causales con un aspecto de su entorno para poder atribuirle la posesión de su concepto, aun cuando nadie estuviera dispuesto a decir que ese individuo es capaz de racionalizar mínimamente su uso del mismo (1986, p. 268). Sin embargo, como estamos viendo, desde el externismo de Burge no podemos marginar los conceptos, exentos de cualquier valor cognitivo para el sujeto, de las explicaciones conceptuales aportadas en su racionalización.

Por último, junto a la propuesta de la teoría desentrecomilladora y a la objeción de Burge consistente en distinguir los conceptos y las explicaciones conceptuales, Bilgrami hace frente a una tercera iniciativa del externismo ortodoxo para impedir la atribución de inconsistencias, iniciativa que propone especificar los conceptos externísticamente individuados de los agentes mediante descripciones metalingüísticas del tipo «artritis es lo que quiera que los expertos denominen artritis» (Bilgrami 1992b, p. 372). Según Bilgrami, todo aquel que ofrezca esa sugerencia estará al mismo tiempo renunciando al externismo ortodoxo, en la medida en que la referencia de los conceptos así especificados estaría completamente determinada por la propia descripción metalingüística (interna al individuo) y no por la referencia del concepto, como defienden los externistas. Sin embargo, consideramos que esta objeción no es insalvable. Ya el propio Putnam en la introducción a (Pessin y Goldberg 1996) ofreció un argumento para responder a un razonamiento muy similar al de Bilgrami expuesto por Searle (1983, pp. 202 ss.). La réplica de Putnam parte de la observación de que las descripciones metalingüísticas de individuos que pertenecen a entornos distintos expresarían contenidos diferentes, por cuanto que ambos individuos tendrían una actitud deferente hacia expertos relevantes de distintas comunidades lingüísticas. La forma de estas descripciones es «artritis es lo que quiera que los expertos de mi comunidad lingüística denominen artritis». Si, por hipótesis, las comunidades lingüísticas difieren en lo que respecta a los usos que establecen para dicho término, y las descripciones metalingüísticas que determinan la referencia expresan contenidos distintos en individuos como Bert y Bert* (puesto que cada una de ellas hace referencia a distintas comunidades lingüísticas), entonces estos sujetos determinarán distintas referencias para los (distintos) conceptos que expresan mediante el término «artritis».

Las conclusiones que podemos establecer hasta el momento confirman que los presupuestos del externismo social de Burge no dan pie tan fácilmente a la 
atribución de pensamientos inconsistentes que Bilgrami recoge en la primera premisa del trilema. Así, para Burge, la mera vinculación causal con los referentes de los conceptos no basta para atribuir la posesión de un concepto a un sujeto si éste carece de una competencia mínima en su dominio, dado lo cual la deuda del externismo social con una teoría de la referencia directa entendida como lo hace Bilgrami ha de matizarse. Por otro lado, Burge admite que los sujetos pueden tener una comprensión parcial de los conceptos junto con una actitud deferente hacia los expertos relevantes de su comunidad lingüística. El hecho de que las descripciones metalingüísticas que recogen dicha actitud deferente puedan estar externísticamente individuadas y, gracias a ello, ser diferentes ante variaciones relevantes del entorno socio-lingüístico, es suficiente para conservar un compromiso robusto con el externismo.

\section{ESENCIALISMO, EXPERIMENTOS MENTALES Y RESTRICCIÓN (C)}

Queda por comentar un aspecto de la descripción de la teoría de la referencia directa que ofrece Bilgrami y es su carácter esencialista. Como vimos, este autor afirma que esta visión de la referencia se encuentra a la base del externismo ortodoxo, así que en la medida en que exista un componente esencialista en la teoría de la referencia directa este componente infectará también las teorías sobre el contenido psicológico que se fundamentan en ella. Para ver la naturaleza y el alcance del esencialismo en el externismo de Burge conviene acercarnos de nuevo a sus textos. Así, Burge nos dice que «el anti-individualismo es una visión sobre las naturalezas de los estados y eventos mentales de carácter representacional. 'Naturaleza' es un término relajado, semi-técnico. El término tradicional es 'esencia'. No tengo nada en contra de las esencias [...] La naturaleza de algo constituye lo que es, sin lo cual no podría ser» (Burge 2007, p. 1).

Burge ofrece una caracterización del término «esencia» de acuerdo con la cual dicho término alude a las condiciones de identidad de un objeto en tanto que objeto de una determinada clase. Así, podríamos denominar a las condiciones de identidad de lo que es ser un (objeto del tipo) $x$ «la esencia de 'x'». Por ejemplo, parece razonable considerar que la esencia de (la clase de objeto que son) los teléfonos móviles consistiría en ser artefactos diseñados para efectuar y recibir llamadas y si un artilugio estuviera diseñado exclusivamente para un fin radicalmente distinto (cicatrizar heridas, pongamos por caso) no parece fácil convencer a alguien de que se trata, en realidad, de una clase de un teléfono móvil. Al entender las esencias o naturalezas de esta manera, en tanto que condiciones de identidad, no sólo creemos ser fieles a las intenciones de Burge sin involucrarle en pesados compromisos ontológicos, sino que con ello logramos entender una de las caracterizaciones usuales de la polémica internismo 
vs. externismo, a saber, la discusión acerca de la localización exclusivamente interna o en parte externa de aquellos factores que determinan esencialmente los contenidos mentales en cuanto que constituyen sus condiciones de identidad. ${ }^{13}$ Es más, el propio Bilgrami asume esta descripción de la problemática en juego, dado que, en su opinión, la determinación externista de los contenidos mentales no responde a una pregunta puramente epistemológica (cómo conocemos los contenidos mentales ajenos), sino que concierne a la naturaleza misma de dichos contenidos (1992b, p. 382). Por otro lado, este autor no sólo acepta hablar de determinación esencial de los contenidos psicológicos, sino que su propia interpretación de las atribuciones mentalistas le lleva a considerar que los conceptos y contenidos mentales cuya posesión adscribimos a los agentes no son meros instrumentos para hacernos inteligible su comportamiento (1992a, pp. $235 \mathrm{ss}$.), lo cual supone que, al menos de forma inespecífica, les reserva un cierto peso ontológico, suficiente para que la pregunta por su naturaleza tenga sentido.

Sin embargo, lo que Bilgrami parece rechazar de forma más clara son las nociones de «naturaleza» o «esencia» entendidas de la manera en que lo hacen los teóricos de la referencia directa, esto es, como aquello que comparten los objetos que forman parte de la extensión de un término y en virtud de lo cual constituyen, precisamente, su extensión. De este modo, los conceptos de naturaleza o esencia estarían estrechamente vinculados con la noción de género natural, pues parece que la esencia es lo que dotaría de identidad a los géneros naturales que constituyen la referencia de los términos que los designan. Es cierto que Burge está comprometido con un «esencialismo» de esta clase, en la medida en que acepta y emplea a su favor (con ciertas precauciones) los experimentos mentales de Putnam, ${ }^{14}$ un autor partidario de esta noción fuerte de género natural que estamos exponiendo. Tanto para Putnam como para Burge, por ejemplo, ser agua es algo que tiene que ver con tener una cierta composición química.

Sin embargo, nos atrevemos a conjeturar que en lo fundamental el externismo de Burge y los experimentos mentales que presenta a su favor pueden defenderse sin apelación a esencia alguna. ${ }^{15}$ Recuérdese al respecto la ironía del autor en (Burge 1979), el documento fundacional del anti-individualismo, cuando declara ignorar si un individuo que rechaza que los acuerdos verbales sean contratos lo hace porque no entiende lo que significa contrato o porque

13 Cf. Wilson 2003, pp. 256-257 y Moya 2004, pp. 153-154.

14 Cf. Burge 1982.

15 Así, es significativo que en aquellas ocasiones en las que Burge expone el contenido del anti-individualismo no emplea los términos « «naturaleza» o 〈esencia» para caracterizar el tipo de variación en el entorno externo al individuo que supondría una variación en sus contenidos mentales. 
está equivocado sobre la esencia de los contratos. Lo relevante para que un experimento mental como el que presenta Burge en ese artículo funcione como experimento a favor del externismo es tomar en consideración una variación en el entorno contrafáctico lo suficientemente importante como para que cualquiera pudiera aceptar que los individuos protagonistas no se refieren a lo mismo mediante proferencias homófonas. En este sentido, nos parece que Tim Crane acierta de pleno cuando afirma que el denominador común de los experimentos mentales que los externistas suelen presentar a su favor es que los individuos que protagonizan dichos experimentos se refieran a algo distinto aunque todo les parezca igual (2001, p. 123).

Adelantamos, además, una segunda conjetura. Pensamos que es significativo el hecho de que Bilgrami no aluda a los experimentos externistas planteados por Putnam en torno a los conceptos de aluminio y molibdeno y de olmo y haya. ${ }^{16} \mathrm{Y}$ creemos que no lo hace porque en este caso Bilgrami estaría de acuerdo en que la referencia de los conceptos y los conceptos mismos involucrados es intuitivamente distinta sin necesidad de apelar a conceptos problemáticos como el de esencia o el de género natural. Más aún, creemos que el análisis que el externismo de Bilgrami podría ofrecer de la situación ideada por Putnam para mostrar la determinación externista de los conceptos de aluminio y molibdeno evidenciaría que existen ciertas tensiones internas a la restricción (C). Para justificar esto, comencemos por reconstruir el experimento mental de Putnam tal y como lo hace Burge al emplearlo a su favor (1989b, p. 316). Imaginemos a un individuo del mundo actual, Paco, quien tiene pensamientos acerca del aluminio. Por ejemplo, sabe que el aluminio es un metal, que se encuentra con más facilidad que el molibdeno y cree, además, que las ollas y las sartenes están hechas de aluminio. Sin embargo, Paco no tiene un dominio experto de ese concepto y, por ejemplo, no sabe en qué se diferencia el aluminio del molibdeno, dos materiales que suponemos indistinguibles a simple vista. Ahora imaginemos a un individuo intrínsecamente idéntico a Paco, llamémosle Paco*, que vive en un entorno distinto, la Tierra Gemela, que difiere de la Tierra en que allí las ollas y sartenes están hechas de molibdeno, un metal mucho más frecuente que el aluminio (tan frecuente respecto a él como lo es en nuestro entorno el aluminio con respecto al molibdeno). Por último, asumamos que los términos «aluminio» $\mathrm{y}$ «molibdeno» tienen sus extensiones permutadas en el lenguaje de Paco* (permaneciendo constantes las propiedades semánticas del resto de los términos lingüísticos) y que, por tanto, mediante el término «aluminio» los hablantes de la Tierra Gemela se refieren al molibdeno y mediante «molibdeno», al aluminio.

Lo que al defensor del externismo à la Burge le interesa preguntar una vez establecido este escenario es lo siguiente: ¿podemos decir que Paco y Paco* 
creen lo mismo cuando dicen «creo que esta olla, como casi todas, está hecha de aluminio»? Atendiendo a las pautas que proporciona la «restricción $(\mathrm{C}) »$ que, recordemos, recoge el contenido del externismo de Bilgrami, parece que al correlacionar las proferencias indéxicas del agente con sus determinantes externos tendremos que admitir que los contenidos de Paco* son diferentes a los de Paco, en tanto que el factor externo que determina de manera relevante las palabras del primero no parece ser de ninguna manera el aluminio, mientras que sí lo es en el caso de Paco. Es decir, que de poner en juego la correlación entre proferencias y determinantes externos que requiere la restricción (C), no podemos decir, como afirman Putnam y Burge, que los pensamientos de Paco y Paco* sean iguales, aun cuando ninguno de ellos sepa en qué difieren. Parece, por tanto, que las exigencias externistas contenidas en la restricción (C) nos invitan a distinguir claramente los contenidos mentales de los protagonistas, extrayendo de este experimento la misma moraleja que el externismo ortodoxo de Putnam y Burge, ${ }^{17}$ algo a lo que en principio parecía resistirse Bilgrami.

A modo de resumen, en esta sección hemos mostrado que, aun dando por válido el retrato que nos ofrece Bilgrami de la teoría causal de la referencia, cabe moderar el alcance que una teoría así tendría en el externismo social de Burge. Además, se han discutido los argumentos que Bilgrami ofrece para detener las acometidas propuestas desde el externismo ortodoxo con el objetivo de evitar su trilema (teoría desentrecomilladora, especificaciones metalingüísticas, distinción conceptos-explicaciones conceptuales). Por último, hemos partido de un experimento mental canónico a favor del planteamiento externista ortodoxo para mostrar, en primer lugar, que el esencialismo ocupa un lugar un tanto secundario en dicha argumentación y, en segundo lugar, que el externismo de Bilgrami no puede evitar atribuir conceptos a la manera del externismo ortodoxo.

\section{Un DILEMA PARA BILGRAMI}

En esta última sección de nuestro artículo vamos a establecer un contraste entre las propuestas de Burge y las de Bilgrami relativas al dominio y la posesión de conceptos por parte de agentes racionales. Con respecto a las ideas del primer autor, ya hemos indicado que para atribuir a un individuo la posesión

17 Y, cabría decir, poniendo en peligro el acceso privilegiado a los propios pensamientos tanto como lo haría peligrar el externismo ortodoxo. En nuestra opinión, que no podemos desarrollar aquí, el hecho de que Paco y Paco* reciban atribuciones de conceptos que en cierto modo «mejoran» su dominio de los mismos no es incompatible (salvo traslados inadvertidos al modo de Boghossian) con el conocimiento que pueden obtener de los contenidos que forman a partir de esos conceptos. 
de un concepto bastaría con que reconociésemos en este sujeto la capacidad de tener pensamientos que lo involucren. Además, según vimos, las premisas que figuran en sus experimentos mentales impiden hacer atribuciones «agresivas», por lo que no podríamos admitir la posesión de ciertos conceptos por parte de aquellas personas respecto de las cuales nadie admitiría que tienen la capacidad de realizar algún tipo de racionalización responsable en la que estuviera involucrado el término (1986, p. 268). Así, parece difícil atribuir a María la posesión de un concepto correspondiente al término «inmarcesible» si lo aplicara al aceite de frenos y además admitiera que lo ha hecho caprichosamente, sin saber en absoluto qué quiere decir mediante el uso de ese adjetivo. Por descontado, nuestras prácticas de atribución mentalista son por entero falibles y nos está permitido rectificar nuestros juicios si descubriéramos que alguien a quien atribuíamos un determinado concepto estaba en realidad bromeando o lo ha aplicado correctamente por casualidad. No obstante, como decimos, para Burge existe un mínimo de competencia a partir de la cual podemos decir que alguien posee un cierto concepto, aunque asocie creencias erróneas con el mismo, siempre y cuando consideremos que el agente es capaz de llevar a cabo una racionalización del término y confíe en los expertos relevantes para rectificar sus creencias. Esa es la clase de competencia básica que mostraría, por ejemplo, Bert, el protagonista del experimento mental de Burge. Por encima de ese nivel que, como decimos, constituye la condición necesaria de la atribución conceptual, podemos encontrar personas con una competencia media en relación con un determinado concepto y, por supuesto, también existe una minoría de individuos, los famosos «expertos», con un alto grado de dominio sobre el concepto relevante. Es importante resaltar a partir de esto que para Burge la competencia en el uso de un concepto admite grados mientras que la posesión de un concepto no. Así, o bien un individuo posee un concepto o bien no lo posee, aunque ello no obsta para que podamos rectificar nuestras atribuciones y resolver que un individuo que parecía poseer un concepto en realidad no lo poseía. Los conceptos son, para Burge, el punto de apoyo a partir del cual somos capaces de establecer caracterizaciones normativas del significado de los términos que los expresan y discutir aquellas caracterizaciones alternativas que se nos presenten. Por poner un ejemplo, un debate en torno al concepto de matrimonio en el cual los participantes asocien distintas creencias a ese concepto y propongan diferentes caracterizaciones de la regla para su uso no tendría sentido en tanto que debate sobre el matrimonio si esta discusión no girara en torno a un mismo concepto.

En lo que respecta a las propuestas de Bilgrami, recordemos que este autor distinguía dos niveles en la atribución de los conceptos. En el nivel agregativo los conceptos se hallan determinados por la totalidad de las creencias que un individuo tiene acerca de dicho concepto. En el nivel local, sin embargo, que un 
individuo posea un concepto es algo que depende de si mantiene acerca de él ciertas creencias, a saber, aquellas creencias que sean relevantes en la localidad donde está situado el sujeto en cuestión. Esto supone, por tanto, que en nuestras prácticas de atribución debemos juzgar cuáles son las creencias pertinentes en función del contexto en el que se lleve a cabo la atribución misma. Así, no es relevante conocer la composición química del amoniaco para atribuir a un agente el deseo de adquirirlo en el supermercado. Su comportamiento queda perfectamente bien explicado sin necesidad de que posea esa clase de información sobre el líquido. Por consiguiente, al contrario que para Burge, no habría un nivel mínimo de competencia a partir del cual se pudiera atribuir a un individuo un determinado concepto. Según Bilgrami, las creencias que el individuo debe poseer para que esa adscripción sea legítima variarán en función de la localidad de la atribución.

Ahora bien, pensamos que la propuesta de Bilgrami puede tener consecuencias un tanto contraintuitivas. Para verlo, imaginemos una variante del experimento mental de Burge en torno al término «artritis» que pone en juego tres localidades entendidas como lo hace Bilgrami. En primer lugar, Bert, el hablante del mundo actual que sufre artritis comenta con su vecina, quien también padece la misma dolencia, la evolución de su enfermedad. En esta primera localidad, ambos protagonistas parecen tener las creencias (muy básicas) que la localidad exige para efectuar la atribución del concepto de artritis. En segundo lugar, Bert acude a su médico, como relata Burge, para exponerle su temor de que la artritis esté afectando a su muslo. Sin embargo, en nuestra variante, el doctor no le corrige la aplicación del término «artritis», sino que se limita a recetarle unas pastillas para el reuma. Para Bilgrami, las creencias relevantes en esta segunda localidad son mucho mayores en número y más sofisticadas y, por tanto, parece que Bert y su médico no compartirían sus conceptos. Imaginemos, por último, una tercera localidad, muy similar a la primera, donde Bert vuelve a encontrarse con su vecina al volver de su visita al médico y entabla con ella una conversación en la que se expresan recíprocamente sus deseos de mejoría en sus padecimientos artríticos. De nuevo, parece que esta localidad «destila», como suele decir Bilgrami, unas creencias relevantes distintas de las creencias relevantes de la segunda localidad y, no obstante, muy similares a las de la primera. En conclusión, Bert «recuperaría» al volver a hablar con su vecina su concepto de artritis, que había «perdido» en la segunda localidad. Pero, ¿hasta qué punto es verosímil hablar de pérdida y recuperación de conceptos? Parece que los presupuestos de la atribución mentalista en función de localidades que Bilgrami pone en juego nos llevan a emplear estos términos. Nótese que esta clase de pérdida no es fruto de un accidente psicológico (un lapsus o una pérdida de memoria), ni de rectificaciones de la clase que Burge admitía. Al contrario que este último, quien admitía variaciones en el grado 
de competencia en el uso de un concepto a partir de la posesión del mismo, Bilgrami disuelve los niveles de dominio en la propia posesión de los conceptos, de forma que un individuo siempre podría «perder» el concepto que posee en la localidad actual (por numerosas y elaboradas que sean las creencias asociadas con el término que expresa el concepto) si se trasladara a otra localidad donde, por ejemplo, entablara una conversación con otro individuo que posee un número mayor de creencias relevantes. A nuestro modo de ver, la distinción entre niveles de competencia que propone Burge es mucho más verosímil que las consecuencias de «pérdida»y «recuperación» de conceptos que parecen desprenderse de la atribución local de Bilgrami, a no ser que concibamos los conceptos como meros instrumentos, como recursos que el intérprete tiene en su mano para hacer inteligible el comportamiento de los agentes en distintas ocasiones. Sin embargo, Bilgrami ha rechazado explícitamente comprometerse con una concepción meramente instrumentalista de los conceptos y contenidos mentales (1992a, pp. 235 ss.). Por esto, podríamos intentar poner a Bilgrami en la tesitura de enfrentarse a un dilema, a saber, o bien suscribe la localidad del contenido con la consiguiente consecuencia indeseada de atribuir «pérdidas» $\mathrm{y}\langle$ recuperaciones» de conceptos, o bien se adhiere a un instrumentalismo en torno a lo mental en cuyas coordenadas dicha consecuencia es inofensiva.

\section{CONCLUSIONES}

A lo largo de este trabajo hemos intentado demostrar que el externismo social desarrollado por Burge tiene recursos suficientes como para no verse afectado por el trilema de Bilgrami. Estos recursos tienen que ver con el hecho de que su deuda con una teoría de la referencia directa, tal y como Bilgrami la entiende, tiene un alcance limitado. Por otro lado, se ha visto que el propio externismo de Bilgrami, recogido en la restricción $(\mathrm{C})$, conserva la tendencia del externismo ortodoxo a realizar atribuciones que completen, sin pasar por alto, el conocimiento que los agentes tienen de sus propios contenidos mentales. Por último, hemos mostrado que el externismo de Bilgrami, cuyas prácticas de atribución se desenvuelven a nivel local, se inclina hacia una concepción meramente instrumentalista de lo mental.

\section{REFERENCIAS BIBLIOGRÁFICAS}

BILGRAMI, A. 1992a: Belief and Meaning: the Unity and Locality of Mental, Cambridge: Blackwell.

BILGRAMI, A. 1992b: «Can externalism be reconcilied with self-knowledge?» en Pessin/ Goldberg 1996, pp. 362-393. 
BILGRAMI, A. 2003: «A trilemma for redeployment». Philosophical Issues, 13, pp. 22-30.

BOGHOSSIAN, P. 1992: «Externalism and inference». Philosophical Issues, 2, pp. $11-28$.

BOGHOSSIAN, P. 1994: «The transparency of mental content». Philosophical Perspectives, 8, pp. 33-50.

BURGE, T. 1979: «Individualism and the mental» en Burge 2007, pp. 100-180.

BURGE, T. 1982: «Other bodies» en Burge 2007, pp. 82-99.

BURGE, T. 1986: «Intellectual norms and foundations of mind» en Burge 2007, pp. 254-274.

BURGE, T. 1989a: «Wherein is language social» en Burge 2007, pp. 275-290.

BURGE, T. 1989b: «Individuation and causation in psychology». En Burge 2007, pp. 316-333.

BURGE, T. 2007: Foundations of Mind, New York: Oxford University Press.

CRANE, T. 2001: Elements of Mind, New York: Oxford University Press.

FODOR, J. A. 1987: Psychosemantics: The problem of meaning in the philosophy of mind, Cambridge, Mass.: MIT Press.

KRIPKE, S. A. 1972: Naming and necessity. Cambridge, Mass.: Harvard University Press.

LOAR, B. 1985: «Social content and psychological content» en Pessin/ Goldberg 1996, pp. 180-190.

MOYA, C. 2004: Filosofía de la mente, Valencia, Universitat de València.

PESSIN, A. Y S. GOLDBERG (eds.) 1996: The twin earth chronicles: twenty years of reflection on Hilary Putnam's «The meaning of meaning», London, Sharpe.

PUTNAM, H. 1975: «The meaning of meaning» en Pessin/ Goldberg 1996, pp. 3-52.

SEARLE, J. 1983: Intentionality: an essay in the philosophy of mind, New York: Cambridge University Press.

SEGAL, G. 2000: A slim book about narrow content, Cambridge, Mass.: MIT Press.

STICH S. Y T. A. WARFIELD (eds.) 2003: The Blackwell guide to philosophy of mind, Malden: Blackwell.

WILSON, R. A. 2003: «Individualism» en Stich/ Warfield 2003, pp. 256-287.

Silvia Andrés Balsera es estudiante de doctorado en el Departamento de Lógica y Filosofía de la Ciencia, Facultad de Filosofía de la Universidad Complutense de Madrid. Becaria FPI por la misma universidad.

\section{Publicaciones recientes}

«El dualismo esquema-contenido en Donald Davidson», Revista Fragmentos (antigua revista Nexo), pendiente de publicación.

Líneas de investigación:

Internismo y externismo en semántica y psicología. También, el relativismo filosófico y el problema del escepticismo sobre el mundo externo.

\section{Dirección electrónica}

s.andres@filos.ucm.es 